\title{
Vitrectomy for Diabetic Macular Edema
}

\author{
Bozho Todorich • Tamer H. Mahmoud
}

Published online: 6 September 2014

(C) Springer Science + Business Media New York 2014

\begin{abstract}
Pars plana vitrectomy represents an important treatment tool in eyes with diabetic macular edema (DME). Vitrectomy primarily promotes resolution of DME by relieving tangential and vitreomacular traction, but has additional benefits of removing depot of VEGF and proinflammatory cytokines, and improving macular oxygen delivery and perfusion. Therefore, vitrectomy has most significant benefit in tractional DME, and potential efficacy in non-tractional DME. The advent of modern vitrectomy with advanced visualization techniques has made macular surgery significantly more safe and improved treatment outcomes. The purpose of the article is to review major studies on the surgical treatment of DME and offer perspectives on strategies to maximize anatomic and functional outcomes of diabetic vitrectomy.
\end{abstract}

Keywords Diabetic macular edema (DME) - Pars plana vitrectomy (PPV) · Vitreomacular traction (VMT) .

Vascular endothelial growth factor (VEGF) - Epiretinal membrane (ERM) · Internal limiting membrane (ILM)

\section{Introduction}

Diabetic retinopathy (DR) is a leading cause of blindness in working-age adults [1]. Diabetic macular edema (DME) is the most common cause of visual morbidity in patients with diabetes mellitus (DM) and affects over 70,000 new patients every year [2,3]. In the Diabetes Control and Complications Trial (DCCT), $27 \%$ of patients developed

B. Todorich · T. H. Mahmoud ( $\square)$

Duke University Eye Center, 2351 Erwin Road, PO Box 3802, Durham, NC 27710, USA

e-mail: thmahmoud@yahoo.com
DME within 9 years of diabetes onset [4], with DME being clinically apparent earlier in older patients with DM [5]. Although spontaneous resolution is described, $24 \%$ of patients with DME and $33 \%$ of patients with centerinvolving DME if untreated will have significant vision loss ( $>15$ ETDRS letters) at 3 years [6]. This number is significantly higher in patients with poor glycemic control.

Multiple biochemical, inflammatory, mechanical, and molecular signaling factors play a role in pathogenesis of DME. Hyperglycemia-mediated accumulation of advanced glycation end products (AGEs) promotes neuro-vascular injury observed in DR [7]. Hypoxia-mediated production of vascular endothelial growth factor (VEGF) results in intracellular signaling with phosphorylation of tight junction proteins leading to increased retinal vascular permeability and breakdown of blood-retina barrier (BRB) [8]. Elevated expression of cell adhesion molecules (ICAM1, VCAM1, PECAM-1, and P-selectin) recruits leukocytes and increases levels of pro-inflammatory cytokines (TNF $\alpha$, angiopoetins, and chemokines) further compromising blood-retinal barrier leading to accumulation of fluid within macula with characteristic cystic changes [9-11]. Therefore, most agents commonly used in treatment of DME today are targeting those main signaling pathways.

With the advent of anti-VEGF therapy over the past decade, multiple large prospective studies have established efficacy of ranibizumab [12, 13•], aflibercept [14], and bevacizumab [11] for treatment of DME. Steroids have been used to treat the inflammatory component of DME either alone $\left[15,16{ }^{\bullet}\right]$ or in combination with anti-VEGF agents or macular laser [17, 18]. Although these agents are widely used for treatment of DME, the discussions of studies establishing their safety and efficacy are beyond the scope of this paper and have been recently reviewed elsewhere [19-24]. 


\section{Vitreomacular Interface in Pathogenesis of DME}

It has been recognized that micro-architecture of posterior hyaloid is significantly different in patients with diabetes compared to non-diabetic patients. Posterior hyaloid in diabetic eyes appears more taut with stronger adherence to the retina, making appearance of posterior vitreous detachment (PVD) rare in these patients. Conversely, complete separation of posterior hyaloid with formation of PVD is associated with decreased rate of DME [25]. With progression of vitreous syneresis, the adherent posterior hyaloid can create anteroposterior vitreomacular traction (VMT) and even shallow macular detachment [26, 27]. Alteration of posterior hyaloid microanatomy with high rate of vitreoschisis may also provide a scaffold for fibrovascular proliferation with formation of contractile lamellae visible on electron microscopy in diabetic eyes [28, 29], and contribute to tangential traction in the macula. Both anteroposterior and tangential traction may promote pathogenesis and refractoriness of DME. Furthermore, adherent posterior hyaloid can serve as a depot for AGEs, and other pro-inflammatory cytokines (i.e., IL-6, sICAM-1) that can drive progression of cystoid macular edema [30-32]. Nasrallah [25] showed that incidence of PVD is lower in patients with DME compared to diabetic patients without DME. Conversely, in one study, spontaneous resolution of DME occurred in $55 \%$ of patients after development of PVD and release of VMT compared to $25 \%$ in eyes without PVD [33]. Collectively, this evidence establishes rationale for use of pars plana vitrectomy (PPV) and removal of posterior hyaloid in treatment of DME.

\section{Vitrectomy and DME}

How does vitrectomy promote resolution of DME? Vitrectomy can be used to relieve macular traction, remove cytokines and pro-angiogenic factors from vitreous, and improve retinal oxygenation and macular perfusion. Ancillary steps during surgery can help resolve DME, such as placement of additional endolaser and steroid depot in the vitreous cavity by virtue of decreasing inflammation and VEGF production [34]. As such, vitrectomy can be effective when patients do not respond or stop responding to intravitreal agents and can help reduce and in some cases eliminate the burden of repeated anti-VEGF injections (Fig. 1). Therefore, vitrectomy remains an important tool in treatment of DME.

Vitrectomy and Tractional DME:

One of the primary mechanisms by which pars plana vitrectomy promotes resolution of DME is by releasing anteroposterior and tangential traction through removal of adherent posterior hyaloid, VMT, epiretinal membrane
(ERM), and internal limiting membrane (ILM) peeling. In a small retrospective study of ten eyes, Lewis and colleagues were first to report the use of PPV in DME eyes with taut posterior hyaloid and traction, which were refractive to macular laser treatment [35]. In this study, all eyes underwent PPV with induction of PVD and lifting of posterior hyaloid, resulting in DME resolution in eight and improved vision in nine out of ten eyes [35]. Subsequently, a number of studies reported overall improved outcomes with PPV for DME associated with VMT [36-38]. One of the largest prospective studies was conducted by DR Clinical Research Network (DRCR.net) and included 87 eyes with DME, VMT, and at least moderate vision loss [39]. The study was conducted at 50 DRCR.net sites in the United States, and surgical techniques were left to the discretion of individual surgeons. Therefore, in addition to PPV, $61 \%$ of patients underwent ERM peeling, $54 \%$ ILM peeling, $40 \%$ panretinal photocoagulation (PRP), and $64 \%$ underwent injection of steroids in the vitreous cavity at the conclusion of the procedure [39]. The main postoperative surgical complications included vitreous hemorrhage, elevated intraocular pressure, retinal detachment ( 3 eyes), and endophthalmitis ( 1 eye). At 6 months of followup, median OCT central subfoveal thickness decreased by 160 microns, and visual acuity improved by 10 or more letters in $38 \%$ of the patients. The anatomic and visual outcomes were maintained at 1-year followup [39]. Overall, these studies provide compelling evidence for use of vitrectomy for cases of DME involving VMT.

\section{ILM Peeling and Tractional DME:}

Does ILM peeling further augment the anatomic and visual outcomes in vitrectomy surgery for DME? Over the past decade, the evolution of modern vitrectomy surgery including small-gage instrumentation and advanced visualization techniques allowed for safe and effective membrane and ILM peels and has facilitated its use in diabetic vitrectomy cases. In particular, ability to stain the ILM during macular surgery with indocyanine green (ICG) and other dyes has allowed for improved visualization of normally transparent tissue, reduced mechanical damage to the retina, and reduced operative time [40, 41]. Although some concerns about toxicity of ICG remain [42-44], the advent of new staining agents such as brilliant blue already in use in Europe offers promise of improved visualization while minimizing RPE and photoreceptor toxicity [45-47]. As a result, since its introduction in 2000, some retina specialists advocate ILM peeling for all vitrectomy cases for DME [48]. ILM may contribute to tangential traction in diabetic patients, and its peeling may relieve that traction. Furthermore, ILM in diabetic patients is thicker and contains higher levels of fibronectin, laminin, and collagen [49, 50], 

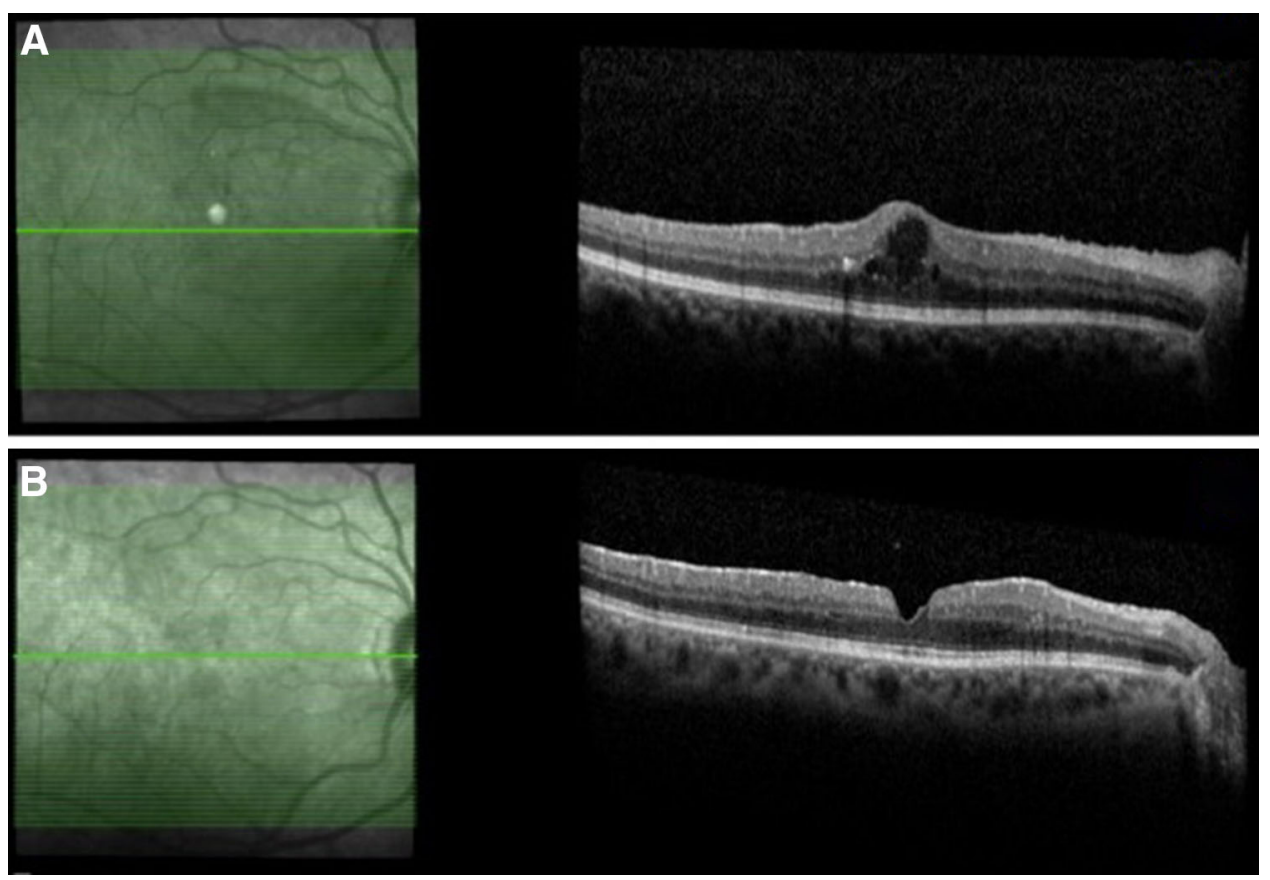

Fig. 1 63-year-old male with history of non-proliferative diabetic retinopathy presented with several months of decreased vision. Visual acuity was 20/70 with central subfoveal cystic changes, distortion of the foveal contour and ERM in the superior macula (a). After multiple intravitreal bevacizumab and ranibizumab injections without improvement, he underwent $23 \mathrm{G}$ pars plana vitrectomy with ERM

raising a possibility of direct toxic effect on the retina. Finally, peeling the ILM prevents recurrences of ERM and ensures removal of all cortical vitreous, which is particularly important in cases of vitreoschisis that have higher risk of retained posterior hyaloid remnants after vitrectomy surgery. The importance of ILM peeling was demonstrated in several clinical studies. Gandorfer [51] and colleagues reported a small series of 12 eyes with diffuse DME, who underwent PPV and ILM peeling. Six of the eyes previously had prior macular laser and two were previously vitrectomized. Intraoperatively light and electron microscopy specimens were obtained and showed thickened posterior hyaloid in most eyes and ILM associated with single layer of fibrous astrocytes [51]. Post-operatively vision improved over 4-12 week period, and 11 out of 12 eyes achieved vision improvement of two lines or better. At mean followup of 16 months, there was no recurrence of macular edema or ERM formation [51]. In another small prospective case series, PPV with ILM peel was performed in eyes with diabetic cystoid macular edema, which was refractory to prior vitrectomy [52]. DME resolved in all eyes with prior vitrectomy after ILM peel with mean central foveal thickness improvement of 553 micron to 221 micron at 4 weeks post-op. In addition, $67 \%$ of eyes experienced visual improvement of at least two lines of vision with no recurrence of DME at mean 17.8 months of and ILM peels, light scatter peripheral endolaser and posterior subtenon kenalog injection. At 6 months post-op, patient had significant anatomic improvement with trace DME, and recovering foveal contour and intact ellipsoid zone (b). He underwent uncomplicated cataract surgery with improved vision to 20/30 and has been stable for over 1 year without injections

followup [52]. Similarly, Gentile et al. [53•] reported two cases of diffuse DME refractive to prior vitrectomy in which a taut residual ILM was noted. Patients underwent repeat PPV with ILM peeling resulting in post-operative improvement in vision and macular edema as noted on OCT imaging. Histologic study of the ILM peel showed cytokeratin and GFAP-positive retinal pigment epithelial cells and astrocytes [53・]. Although further prospective studies are warranted, ILM peeling may represent an important adjunct in diabetic PPV cases and can further augment the visual and anatomic outcomes in patients with DME.

\section{Vitrectomy and Non-tractional DME}

The collateral, non-mechanical benefits of PPV in eyes with DME also include increased oxygenation to the macula $[54,55]$ and removal of cytokine and chemokine depot that promotes breakdown of BRB (i.e., VEGF). Removal of VEGF and pro-inflammatory cytokines may promote integrity of $\mathrm{BRB}$, facilitating removal of fluid within the macula by the RPE pump and suppression of inflammation. In some studies, oxygen tension in the vitreous cavity following vitrectomy surgery increases tenfold [56], with a secondary benefit of increased retinal perfusion. This alone may further result in long-term decrease in 

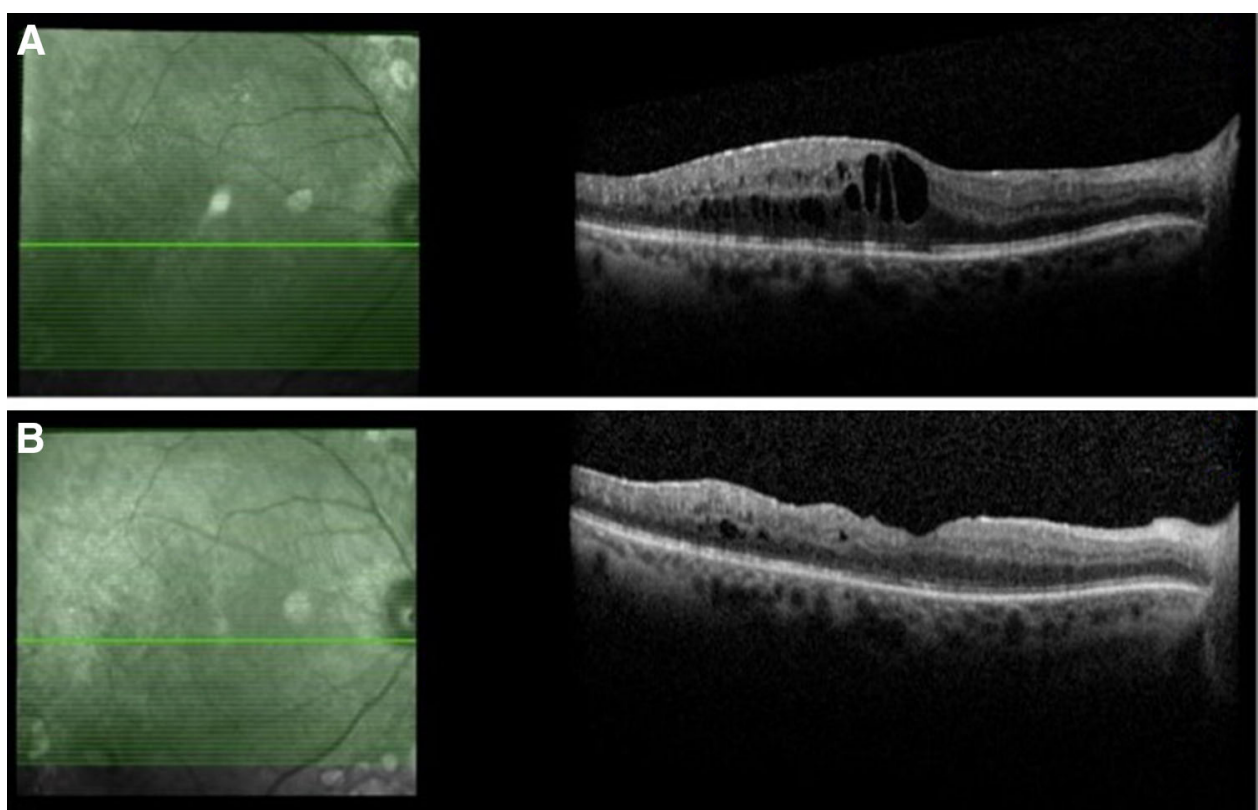

Fig. 2 62-year-old male with history of proliferative diabetic retinopathy and diabetic macular edema presented with non-clearing vitreous hemorrhage. He underwent PPV with induction of PVD and endolaser, and initially experienced improvement in vision without DME. However, 6 months later, he developed worsening cystic changes in the temporal macula consistent with DME with 20/100 vision (a). Multiple injections of bevacizumab, ranibizumab, and

VEGF levels providing sustained suppression of DME. Therefore, vitrectomy may be effective in DME eyes without VMT. In a small prospective randomized trial, 25 eyes with diffuse chronic DME without VMT or macular ischemia underwent PPV, induction of posterior hyaloid, and ILM peeling [57]. Most eyes had prior macular laser, and the untreated eyes served as a control. At 6 months, vitrectomized eyes demonstrated improved macular thickness and visual acuity, suggesting superiority of PPV with ILM peeling for chronic diffuse DME to observation alone [57]. Our experience has been that treatment outcomes in select cases of recurrent, non-tractional DME can be augmented with vitrectomy surgery and ILM peeling (Fig. 2). A number of subsequent studies demonstrated much less favorable outcomes [58-62, 63••, 64-67], and a recent meta-analysis integrating the collective data of these studies showed rather modest overall visual and anatomic benefit of PPV for non-tractional DME [68]. Therefore, further prospective studies are needed to determine which patients with non-tractional DME would benefit most from diabetic vitrectomy.

\section{Patient Selection and Technical Considerations}

Not infrequently patients with recalcitrant DME will fail to improve after vitrectomy or rarely continue to lose vision. posterior subtenons kenalog were given without clear improvement in vision or OCT appearance. For that reason, he underwent $25 \mathrm{G}$ pars plana vitrectomy, ILM peel, and intravitreal avastin. Two months following second surgery vision improved to 20/50 with decreased intraretinal fluid, subfoveal thickness and improved foveal contour (b). Vision gradually improved to $20 / 40$ and was stable for 1 year without injections

How can patient selection of surgical candidates be optimized to maximize visual outcomes? To help answer this question, DRCR.net prospectively enrolled 241 eyes at 50 clinical sites that underwent vitrectomy surgery for DME [69]. Multivariate analysis was performed on 20 pre- and intra-operative factors associated with visual and anatomic outcomes at 6 months post-surgery [69]. Almost three quarters of eyes had prior treatment for DME with most receiving macular laser or steroids, and $71 \%$ of eyes had vitreomacular interface abnormalities or traction. The surgical procedure was performed at the discretion of the individual investigator, and included PPV with lifting of posterior hyaloid in almost all eyes, ERM peeling in $61 \%$ eyes, removal of ILM in $64 \%$ eyes, additional laser in $54 \%$, and use of corticosteroids (intravitreal, subtenons, peribulbar, or subconjunctival) at the completion of vitrectomy in $68 \%$ of eyes [69]. At 6 months, the mean central foveal thickness significantly decreased from 412 to 278 microns, but overall visual acuity did not change and remained at mean 20/80 Snellen. The factors significantly associated with anatomic improvement included worse baseline vision and central subfoveal thickness on OCT, presence of vitreoretinal abnormalities, and removal of ILM [69]. However, the visual acuity improvement was associated only with worse pre-operative visual acuity and ERM peeling [69]. Overall, these results suggest that visual 
improvement does not always correlate to anatomic improvement [70] and that patients with worse pre-op vision requiring ERM peeling were best surgical candidates for visual improvement after DME vitrectomy. Interestingly, in DRCR.net study, neither ILM peeling nor clinician assessment of the posterior hyaloid was significantly associated with the visual outcomes even though they were in multiple other studies [69]. Although this study is important, it is significantly limited by a number of factors. Absence of a control group, significant heterogeneity and lack of standardized surgeons' surgical approaches, and differing baseline characteristics of patients including variable chronicity of underlying DME reduce the impact of the study conclusions.

Even though the risks of modern vitrectomy surgery are small, the risk of cataract progression, low but not insignificant rate of endophthalmitis and retinal detachment, and post-operative IOP spikes need to be weighed against the potential benefits of PPV in eyes with DME. Furthermore, loss of vitreous depot will affect pharmacokinetics of intravitreal injectables such as anti-VEGF agents, significantly shortening their half-life and may limit their efficacy for DME in vitrectomized eyes [71]. As a result, most surgeons currently employ vitrectomy relatively late in the course of DME treatment, often after multiple other therapies have been exhausted.

So, what options are there if vitrectomy also fails to improve vision or if DME recurs at some time after surgery? Surgical failure in our experience can come from at least two important factors; one is incomplete removal of posterior hyaloid and second is irreversible loss of outer retinal layers (ellipsoid zone and external limiting membrane) as a result of chronicity of DME. In our experience, induction of PVD with complete removal of posterior vitreous cortex is essential to achieving good surgical outcome. Leaving a sheet of hyaloid or vitreous remnants can serve as a nidus for proliferation of epithelial cells, astrocytes, and fibroblasts that promote development or recurrence of ERM and consequently DME. Not infrequently, it is difficult to separate the vitreous from the nerve and the macula and induce PVD in diabetic eyes. Use of bimanual techniques with use of lighted pick can facilitate the separation of posterior hyaloid and may be helpful in tough cases (Fig. 3). We also routinely utilize dilute triamcinolone to visualize posterior hyaloid, detect vitreoschisis and pockets of adherent cortical vitreous remaining after PVD induction, and take meticulous care to remove all visible hyaloid. Even so, some authors argue that only ILM peeling can guarantee complete removal of all residual vitreous remnants. We do advocate peeling ILM for cases of recalcitrant DME, and use visualization techniques such as ICG staining in almost every case. Chronic DME is associated with loss of photoreceptor outer segments, retinal thinning, and loss of retinal function. The mechanism by which this occurs is likely multifactorial and includes ischemia, mechanical stress from intercellular fluid accumulation or traction, neuro-inflammatory cytotoxicity, separation of photoreceptors from RPE, and choroid by subretinal fluid or choroidal thinning [72, 73]. The advent of SD OCT imaging in 2006 significantly enhanced our ability to detect outer retinal changes and correlate them to visual loss in variety of retinal conditions, including DME. In one study involving 69 eyes that underwent vitrectomy for DME, the integrity of the photoreceptor inner and outer segment (IS/OS) line was investigated by OCT at 6 months post-op [74]. Final visual acuity correlated well to loss of IS/OS junction integrity on OCT with eyes having loss of IS/OS junction experiencing lower percentage of visual improvement following vitrectomy [74]. Similar correlation was found in few non-surgical studies in patients with DME $\left[7^{\bullet}, 76\right]$. Even though

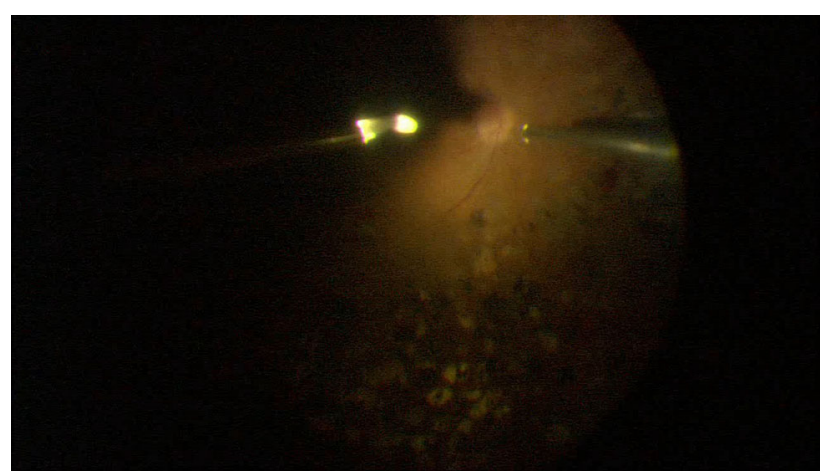

Fig. 3 Patient with proliferative diabetic retinopathy and DME underwent pars plana vitrectomy, membrane, and ILM peeling. In this intraoperative image, lighted pick is used to engage and separate adherent posterior hyaloid and facilitate intraoperative PVD induction

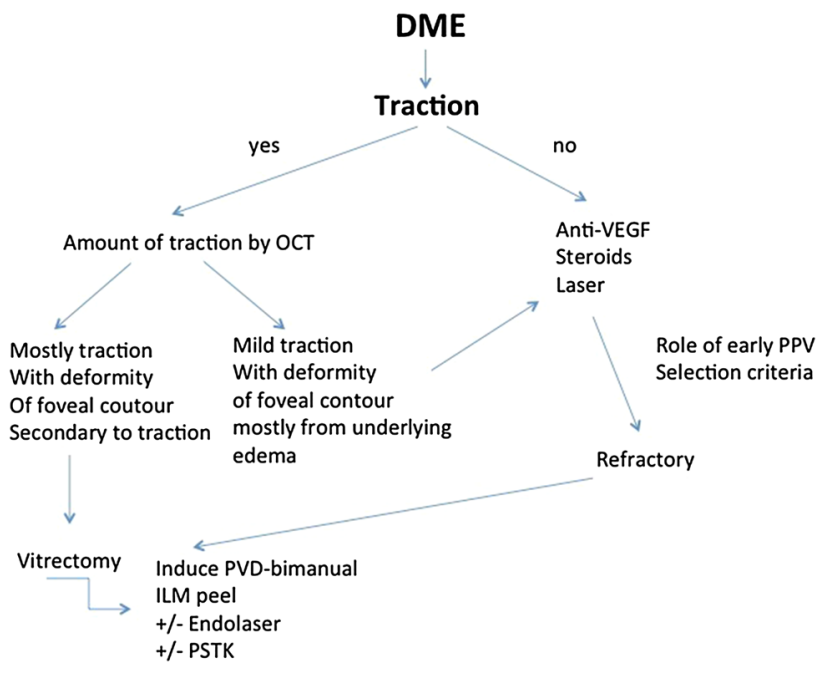

Fig. 4 Proposed algorithm for surgical treatment of DME 
assessment of photoreceptor anatomy by SD OCT may be important prognostic indicator, it is certainly not always perfectly predictive. Shadowing artifacts from dense ERM, extensive cystoid macular edema, vitreous opacities, or vitreomacular interface abnormalities may pre-operatively give impression of disruption of ellipsoid zone despite intact photoreceptor layer observed post-op. Conversely, in our experience, even SD OCT may not show residual traction well in post-vitrectomy diabetic eyes, which has important prognostic implications in cases of recurrent DME. Finally, recent reports indicate that subfoveal choroidal thickness on EDI SD OCT imaging is decreased in patients with proliferative DR and DME [77]. We have recently demonstrated that subfoveal choroidal thickness in eyes with DME decreases with anti-VEGF therapy, but this does not correlate with anatomic and functional improvement [78••]. This all begs the question of the value of early PPV in the management of DME, prior to irreversible damage at the levels of the outer layers by chronic refractory DME.

\section{Conclusion}

Vitrectomy remains an important tool in treatment of select cases of DME by virtue of removing traction, improving macular oxygenation, removing VEGF and pro-inflammatory cytokines, and allowing for placement of additional endolaser and steroids. Modern vitrectomy surgery with small-gage systems, advanced visualization for membrane and ILM peeling, and bimanual techniques offer promise of safe surgery with improved efficacy. As a result, vitrectomy is primarily indicated in DME cases involving ERM and VMT, with less benefits for refractory non-tractional DME (Fig. 4). Further prospective studies are needed to define the role of early PPV and its application for nontractional DME.

Disclosure Bozho Todorich has no reported conflict of interest. Tamer H. Mahmoud serves as a consultant for Alcon Alimera, Allergan, and the FDA.

Human and Animal Rights and Informed Consent This article does not contain any studies with human or animal subjects performed by any of the authors.

\section{References}

1. Antonetti DA, Klein R, Gardner TW. Diabetic retinopathy. The New England journal of medicine. 2012;366(13):1227-39.

2. Coscas G, Cunha-Vaz J, Loewenstein A, Soubrane G. In, "Macular Edema: Practical Approach". Basel: Karger; 2010.

3. Bresnick GH. Diabetic macular edema. A review. Ophthalmology. 1986;93(7):989-97.
4. Progression of retinopathy with intensive versus conventional treatment in the Diabetes Control and Complications Trial. Diabetes Control and Complications Trial Research Group. Ophthalmology. 1995;102(4):647-61.

5. Klein R KB, Moss SE. The epidemiology of ocular problems in diabetes mellitus. In: Stephen SF, editor. Ocular problems in diabetes mellitus. Boston: Blackwell Scientific Publications; 1991.

6. Photocoagulation for diabetic macular edema. Early Treatment Diabetic Retinopathy Study report number 1. Early Treatment Diabetic Retinopathy Study research group. Arch Ophthalmol. 1985;103(12):1796-806.

7. Kim W, Hudson BI, Moser B, et al. Receptor for advanced glycation end products and its ligands: a journey from the complications of diabetes to its pathogenesis. Ann N Y Acad Sci. 2005; 1043:553-61.

8. Antonetti DA, Barber AJ, Hollinger LA, Wolpert EB, Gardner TW. Vascular endothelial growth factor induces rapid phosphorylation of tight junction proteins occludin and zonula occluden 1. A potential mechanism for vascular permeability in diabetic retinopathy and tumors. J Biol Chem. 1999;274(33):23463-7.

9. Rangasamy S, McGuire PG, Das A. Diabetic retinopathy and inflammation: novel therapeutic targets. Middle East African journal of ophthalmology. 2012;19(1):52-9.

10. Klaassen I, Van Noorden CJ, Schlingemann RO. Molecular basis of the inner blood-retinal barrier and its breakdown in diabetic macular edema and other pathological conditions. Progress in retinal and eye research. 2013;34:19-48.

11. Arevalo JF, Fromow-Guerra J, Quiroz-Mercado H, et al. Primary intravitreal bevacizumab (Avastin) for diabetic macular edema: results from the Pan-American Collaborative Retina Study Group at 6-month follow-up. Ophthalmology. 2007;114(4):743-50.

12. Nguyen QD, Brown DM, Marcus DM, et al. Ranibizumab for diabetic macular edema: results from 2 phase III randomized trials: RISE and RIDE. Ophthalmology. 2012;119(4):789-801.

13. - Brown DM, Nguyen QD, Marcus DM et al. Long-term outcomes of ranibizumab therapy for diabetic macular edema: the 36-month results from two phase III trials: RISE and RIDE. Ophthalmology. 2013;120(10):2013-22. This study summarizes long-term outcomes of two-landmark trials, RISE and RIDE for treatment of Diabetic Macular Edema (DME).

14. Do DV, Nguyen QD, Boyer D, et al. One-year outcomes of the da Vinci Study of VEGF Trap-Eye in eyes with diabetic macular edema. Ophthalmology. 2012;119(8):1658-65.

15. Haller JA, Bandello F, Belfort R Jr, et al. Randomized, shamcontrolled trial of dexamethasone intravitreal implant in patients with macular edema due to retinal vein occlusion. Ophthalmology. 2010;117(6):1134-46 e1133.

16. • Haller JA, Kuppermann BD, Blumenkranz MS et al. Randomized controlled trial of an intravitreous dexamethasone drug delivery system in patients with diabetic macular edema. Arch ophthalmol. 2010;128(3):289-96. This study established safety and efficacy of OZURDEX (intravitreous dexamethasone drug delivery system) for treatment of diabetic macular edema.

17. Diabetic Retinopathy Clinical Research N, Elman MJ, Aiello LP, et al. Randomized trial evaluating ranibizumab plus prompt or deferred laser or triamcinolone plus prompt laser for diabetic macular edema. Ophthalmology. 2010;117(6):1064-77 e1035.

18. Sutter FK, Simpson JM, Gillies MC. Intravitreal triamcinolone for diabetic macular edema that persists after laser treatment: three-month efficacy and safety results of a prospective, randomized, double-masked, placebo-controlled clinical trial. Ophthalmology. 2004;111(11):2044-9.

19. Boyer DS, Hopkins JJ, Sorof J, Ehrlich JS. Anti-vascular endothelial growth factor therapy for diabetic macular edema. Therapeutic advances in endocrinology and metabolism. 2013;4(6):151-69. 
20. Stefanini FR, Badaro E, Falabella P, Koss M, Farah ME, Maia M. Anti-VEGF for the Management of Diabetic Macular Edema. Journal of immunology research. 2014;2014:632307.

21. Zhang X, Wang N, Schachat AP, Bao S, Gillies MC. Glucocorticoids: structure, signaling and molecular mechanisms in the treatment of diabetic retinopathy and diabetic macular edema. Curr Mol Med. 2014;14(3):376-84.

22. Al Dhibi HA, Arevalo JF. Clinical trials on corticosteroids for diabetic macular edema. World journal of diabetes. 2013;4(6): 295-302.

23. Bandello F, Casalino G, Loewenstein A, Goldstein M, Pelayes D, Battaglia Parodi M. Pharmacological approach to diabetic macular edema. Ophthalmic Res. 2014;51(2):88-95.

24. Abouammoh MA. Ranibizumab injection for diabetic macular edema: meta-analysis of systemic safety and systematic review. Canadian journal of ophthalmology. Journal canadien d'ophtalmologie. 2013;48(4):317-23.

25. Nasrallah FP, Jalkh AE, Van Coppenolle F, et al. The role of the vitreous in diabetic macular edema. Ophthalmology. 1988; 95(10):1335-9.

26. Kaiser PK, Riemann CD, Sears JE, Lewis H. Macular traction detachment and diabetic macular edema associated with posterior hyaloidal traction. Am J Ophthalmol. 2001;131(1):44-9.

27. Massin P, Duguid G, Erginay A, Haouchine B, Gaudric A. Optical coherence tomography for evaluating diabetic macular edema before and after vitrectomy. Am J Ophthalmol. 2003;135(2):169-77.

28. Faulborn J, Bowald S. Microproliferations in proliferative diabetic retinopathy and their relationship to the vitreous: corresponding light and electron microscopic studies. Graefe's Archive Clin Exp Ophthalmol (Albrecht von Graefes Archiv fur klinische und experimentelle Ophthalmologie). 1985;223(3):130-8.

29. Jumper JM, Embabi SN, Toth CA, McCuen BW II, Hatchell DL. Electron immunocytochemical analysis of posterior hyaloid associated with diabetic macular edema. Retina. 2000;20(1): 63-8.

30. Christoforidis JB, D'Amico DJ. Surgical and other treatments of diabetic macular edema: an update. Int Ophthalmol Clin. 2004;44(1):139-60.

31. Funatsu H, Yamashita H, Sakata K, et al. Vitreous levels of vascular endothelial growth factor and intercellular adhesion molecule 1 are related to diabetic macular edema. Ophthalmology. 2005;112(5):806-16.

32. Funatsu H, Yamashita H, Ikeda T, Mimura T, Eguchi S, Hori S. Vitreous levels of interleukin-6 and vascular endothelial growth factor are related to diabetic macular edema. Ophthalmology. 2003;110(9):1690-6.

33. Hikichi T, Fujio N, Akiba J, Azuma Y, Takahashi M, Yoshida A. Association between the short-term natural history of diabetic macular edema and the vitreomacular relationship in type II diabetes mellitus. Ophthalmology. 1997;104(3):473-8.

34. Mehta AN,Aaberg Jr TM, Mahmoud TH, Duesbery N. Differences in intraocular levels of vascular endothelial growth factor in diffuse vs focal diabetic macular edema. Invest Ophthalmol Vis Sci. ARVO E-Abstract 5044. 2010.

35. Lewis H, Abrams GW, Blumenkranz MS, Campo RV. Vitrectomy for diabetic macular traction and edema associated with posterior hyaloidal traction. Ophthalmology. 1992;99(5):753-9.

36. Harbour JW, Smiddy WE, Flynn HW Jr, Rubsamen PE. Vitrectomy for diabetic macular edema associated with a thickened and taut posterior hyaloid membrane. Am J Ophthalmol. 1996;121(4):405-13.

37. Pendergast SD, Hassan TS, Williams GA, et al. Vitrectomy for diffuse diabetic macular edema associated with a taut premacular posterior hyaloid. Am J Ophthalmol. 2000;130(2):178-86.

38. Recchia FM, Ruby AJ, Carvalho Recchia CA. Pars plana vitrectomy with removal of the internal limiting membrane in the treatment of persistent diabetic macular edema. Am J Ophthalmol. 2005;139(3):447-54.

39. Diabetic Retinopathy Clinical Research Network Writing C, Haller JA, Qin H et al. Vitrectomy outcomes in eyes with diabetic macular edema and vitreomacular traction. Ophthalmology, 2010;117(6):1087-93 e1083.

40. Kadonosono K, Itoh N, Uchio E, Nakamura S, Ohno S. Staining of internal limiting membrane in macular hole surgery. Arch Ophthalmol. 2000;118(8):1116-8.

41. Burk SE, Da Mata AP, Snyder ME, Rosa RH Jr, Foster RE. Indocyanine green-assisted peeling of the retinal internal limiting membrane. Ophthalmology. 2000;107(11):2010-4.

42. Kusaka S, Oshita T, Ohji M, Tano Y. Reduction of the toxic effect of indocyanine green on retinal pigment epithelium during macular hole surgery. Retina. 2003;23(5):733-4.

43. Ho JD, Chen HC, Chen SN, Tsai RJ. Reduction of indocyanine green-associated photosensitizing toxicity in retinal pigment epithelium by sodium elimination. Arch Ophthalmol. 2004;122(6):871-8.

44. Engelbrecht NE, Freeman J, Sternberg P Jr, et al. Retinal pigment epithelial changes after macular hole surgery with indocyanine green-assisted internal limiting membrane peeling. Am J Ophthalmol. 2002;133(1):89-94.

45. Hisatomi T, Enaida H, Matsumoto H, et al. Staining ability and biocompatibility of brilliant blue G: preclinical study of brilliant blue $\mathrm{G}$ as an adjunct for capsular staining. Arch Ophthalmol. 2006;124(4):514-9.

46. Enaida H, Hisatomi T, Hata Y, et al. Brilliant blue G selectively stains the internal limiting membrane/brilliant blue G-assisted membrane peeling. Retina. 2006;26(6):631-6.

47. Enaida H, Hisatomi T, Goto Y, et al. Preclinical investigation of internal limiting membrane staining and peeling using intravitreal brilliant blue G. Retina. 2006;26(6):623-30.

48. Hassan TS. Vitrectomy for diabetic macular edema. Rev Ophthalmol. 2006.

49. Kohno T, Sorgente N, Goodnight R, Ryan SJ. Alterations in the distribution of fibronectin and laminin in the diabetic human eye. Invest Ophthalmol Vis Sci. 1987;28(3):515-21.

50. Ljubimov AV, Burgeson RE, Butkowski RJ, et al. Basement membrane abnormalities in human eyes with diabetic retinopathy. The journal of histochemistry and cytochemistry : official journal of the Histochemistry Society. 1996;44(12):1469-79.

51. Gandorfer A, Messmer EM, Ulbig MW, Kampik A. Resolution of diabetic macular edema after surgical removal of the posterior hyaloid and the inner limiting membrane. Retina. 2000;20(2):126-33.

52. Kimura T, Kiryu J, Nishiwaki H, et al. Efficacy of surgical removal of the internal limiting membrane in diabetic cystoid macular edema. Retina. 2005;25(4):454-61.

53. - Gentile RC, Milman T, Eliott D, Romero JM, McCormick SA. Taut internal limiting membrane causing diffuse diabetic macular edema after vitrectomy: clinicopathological correlation. Ophthalmologica. (Journal international d'ophtalmologie. International journal of ophthalmology. Zeitschrift fur Augenheilkunde). 2011;226(2):64-70. This study reported 2 cases of DME undergoing vitrectomy with ILM peel. Histology of removed ILM showed an inner monolayer of cytokeratin-positive (retinal pigment epithelial cells) and/or glial fibrillary acidic protein-positive cells with smooth muscle actin immunoreactivity.

54. Stefansson E, Landers MB 3rd, Wolbarsht ML. Increased retinal oxygen supply following pan-retinal photocoagulation and vitrectomy and lensectomy. Trans Am Ophthalmol Soc. 1981;79:307-34.

55. Stefansson E, Novack RL, Hatchell DL. Vitrectomy prevents retinal hypoxia in branch retinal vein occlusion. Invest Ophthalmol Vis Sci. 1990;31(2):284-9. 
56. Holekamp NM, Shui YB, Beebe DC. Vitrectomy surgery increases oxygen exposure to the lens: a possible mechanism for nuclear cataract formation. Am J Ophthalmol. 2005;139(2):302-10.

57. Stolba U, Binder S, Gruber D, Krebs I, Aggermann T, Neumaier B. Vitrectomy for persistent diffuse diabetic macular edema. Am J Ophthalmol. 2005;140(2):295-301.

58. Yanyali A, Horozoglu F, Celik E, Ercalik Y, Nohutcu AF. Pars plana vitrectomy and removal of the internal limiting membrane in diabetic macular edema unresponsive to grid laser photocoagulation. Eur J Ophthalmol. 2006;16(4):573-81.

59. Kumar A, Sinha S, Azad R, Sharma YR, Vohra R. Comparative evaluation of vitrectomy and dye-enhanced ILM peel with grid laser in diffuse diabetic macular edema. Graefe's Arch Clin Exp Ophthalmol (Albrecht von Graefes Archiv fur klinische und experimentelle Ophthalmologie).2007;245(3):360-8.

60. Yanyali A, Nohutcu AF, Horozoglu F, Celik E. Modified grid laser photocoagulation versus pars plana vitrectomy with internal limiting membrane removal in diabetic macular edema. Am J Ophthalmol. 2005;139(5):795-801.

61. Patel JI, Hykin PG, Schadt M, et al. Diabetic macular oedema: pilot randomised trial of pars plana vitrectomy vs macular argon photocoagulation. Eye. 2006;20(8):873-81.

62. Thomas D, Bunce C, Moorman C, Laidlaw DA. A randomised controlled feasibility trial of vitrectomy versus laser for diabetic macular oedema. The British journal of ophthalmology. 2005;89(1):81-6.

63. •- Hoerauf H, Bruggemann A, Muecke M et al. Pars plana vitrectomy for diabetic macular edema. Internal limiting membrane delamination vs posterior hyaloid removal. A prospective randomized trial. Graefe's Arch Clin Exp Ophthal (Albrecht von Graefes Archiv fur klinische und experimentelle Ophthalmologie) 2011;249(7):997-1008. This small prospective randomized clinical trial showed that pars plana vitrectomy with PVD induction with or without ILM peeling does not appear to confer visual benefit in eyes with DME without vitreo-macular traction.

64. Bahadir M, Ertan A, Mertoglu O. Visual acuity comparison of vitrectomy with and without internal limiting membrane removal in the treatment of diabetic macular edema. Int Ophthalmol. 2005;26(1-2):3-8.

65. Bardak Y, Cekic O, Tig SU. Comparison of ICG-assisted ILM peeling and triamcinolone-assisted posterior vitreous removal in diffuse diabetic macular oedema. Eye. 2006;20(12):1357-9.

66. Doi N, Sakamoto T, Sonoda Y et al. Comparative study of vitrectomy versus intravitreous triamcinolone for diabetic macular edema on randomized paired-eyes. Graefe's Arch Clin Exp Ophthalmol (Albrecht von Graefes Archiv fur klinische und experimentelle Ophthalmologie) 2012;250(1):71-8.

67. Figueroa MS, Contreras I, Noval S. Surgical and anatomical outcomes of pars plana vitrectomy for diffuse nontractional diabetic macular edema. Retina. 2008;28(3):420-6.
68. Simunovic MP, Hunyor AP, Ho IV. Vitrectomy for diabetic macular edema: a systematic review and meta-analysis. Canadian journal of ophthalmology. Journal canadien d'ophtalmologie. 2014;49(2):188-95.

69. Flaxel CJ, Edwards AR, Aiello LP, et al. Factors associated with visual acuity outcomes after vitrectomy for diabetic macular edema: diabetic retinopathy clinical research network. Retina. 2010;30(9):1488-95.

70. Mehta MU, Tewari A, Mahmoud TH. Structural and functional outcome of PPV with ILM peeling for refractory diabetic macular edema. Invest Ophthalmol Vis Sci. (ARVO E-Abstract 993). 2011.

71. Christoforidis JB, Williams MM, Wang J, et al. Anatomic and pharmacokinetic properties of intravitreal bevacizumab and ranibizumab after vitrectomy and lensectomy. Retina. 2013;33(5): 946-52.

72. Lardenoye CW, Probst K, DeLint PJ, Rothova A. Photoreceptor function in eyes with macular edema. Invest Ophthalmol Vis Sci. 2000;41(12):4048-53.

73. Murakami T, Yoshimura N. Structural changes in individual retinal layers in diabetic macular edema. Journal of diabetes research. 2013;2013:920713.

74. Sakamoto A, Nishijima K, Kita M, Oh H, Tsujikawa A, Yoshimura N. Association between foveal photoreceptor status and visual acuity after resolution of diabetic macular edema by pars plana vitrectomy. Graefe's Arch Clin Exp Ophthalmol (Albrecht von Graefes Archiv fur klinische und experimentelle Ophthalmologie) 2009;247(10):1325-30.

75. - Maheshwary AS, Oster SF, Yuson RM, Cheng L, Mojana F, Freeman WR. The association between percent disruption of the photoreceptor inner segment-outer segment junction and visual acuity in diabetic macular edema. Am J Ophthalmol. 2010;150(1):63-7 e61 (2010). This study showed correlation between photoreceptor inner segment/outer segment (IS/OS) junction disruption and visual loss in patients with diabetic macular edema.

76. Otani T, Yamaguchi Y, Kishi S. Correlation between visual acuity and foveal microstructural changes in diabetic macular edema. Retina. 2010;30(5):774-80.

77. Unsal E, Eltutar K, Zirtiloglu S, Dincer N, Ozdogan Erkul S, Gungel H. Choroidal thickness in patients with diabetic retinopathy. Clinical ophthalmology. 2014;8:637-42.

78. •• Yiu G MV, Chiu SJ, Mahmoud TH. Effect of anti-vascular endothelial growth factor therapy on choroidal thickness in diabetic macular edema. Am J Ophthalmol, (in press) (2014). This study showed that subfoveal choroidal thickness decreases in response to anti-VEGF therapy in eyes DME; this has potentially significant treatment implications for DME. 